\title{
Le double-jeu de la protéine TIP47
}

> Un chaînon manquant dans la compréhension

Alain Pauloin, Michèle Ollivier-Bousquet, Éric Chanat

des mécanismes de transport des récepteurs du mannose 6-phosphate vient d'être récemment découvert à la suite de l'identification de la protéine TIP47. Cette protéine, en association avec Rab9-GTP, assure le retour de ces récepteurs des endosomes tardifs vers le réseau trans-golgien. Curieusement, la même protéine, baptisée PP17b, avait été décrite comme une protéine placentaire il y a vingt ans et, plus récemment, comme un marqueur d'évolution du cancer du col de l'utérus. Non seulement la séquence de la PP17b/TIP47 présente une forte homologie avec celles de l'adipophiline et des périlipines, protéines connues pour participer au trafic intracellulaire des gouttelettes lipidiques, mais la PP17b/TIP47 est aussi associée à ces dernières. Comment cette protéine peut-elle participer en même temps à des processus aussi différents que le transport du récepteur du mannose 6-phosphate et la formation et/ou le transport des gouttelettes lipidiques? Une première hypothèse est proposée. <

Destinées aux lysosomes, les hydrolases nouvellement synthétisées dans le réticulum endoplasmique sont transportées vers l'appareil de Golgi. Elles y acquièrent une modification post-traductionnelle spécifique: I'ajout de résidus terminaux mannose 6-phosphate (M6P) sur un groupement oligosaccharidique [1]. Parvenues au réseau trans-golgien, ces enzymes sont reconnues spécifiquement par des protéines transmembranaires appelées récepteurs du M6P [2]. Deux récepteurs ont été décrits, un récepteur court $(45 \mathrm{kDa})$, appelé récepteur dépendant des cations (CD) - les cations divalents étant nécessaires à la liaison du ligand sur son récepteur - et un récepteur long $(300 \mathrm{kDa})$, appelé récepteur indépendant des cations (CI) [3]. Les deux types de récepteurs du M6P transportent leurs ligands du réseau trans-golgien vers les com-

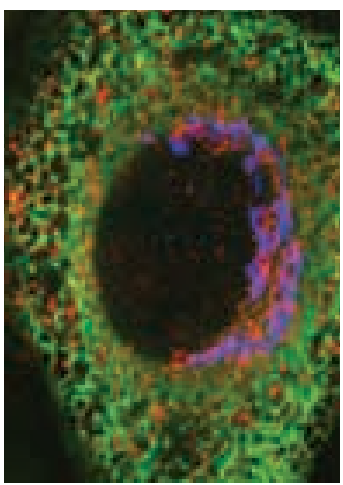

partiments prélysosomiaux (endosomes tardifs) où ils les libè-
Unité de Génomique et physiologie de la lactation, Inra, 78352 Jouy-en-Josas

Cedex, France.

pauloin@jouy.inra.fr rent en réponse au $\mathrm{pH}$ acide local. Ils sont ensuite redirigés vers le réseau trans-golgien pour un nouveau cycle de transport (Figure 1).

Si les mécanismes de reconnaissance moléculaire qui gouvernent le ciblage et le transport des enzymes lysosomiales à la sortie du réseau trans-golgien commencent à être relativement bien connus, ceux qui sont impliqués dans le retour du récepteur du M6P vers ce compartiment restaient à mettre en évidence. Une bonne partie du voile a été levé par le groupe de S. Pfeffer qui a découvert une protéine spécifiquement responsable de cette fonction, TIP47 (tail-interacting protein of $47 \mathrm{kDa}$ ) [4]. Curieusement, la séquence de TIP47 est $100 \%$ similaire à celle de la PP17b (placental protein $17^{\text {th }}$ ), une version longue de la protéine PP17a, une protéine cytosolique placentaire de $38 \mathrm{kDa}$ isolée dès 1983 mais dont le rôle physiologique reste toujours à découvrir $[5,6]$. Deux autres variants protéiques ont aussi été mis en évidence: $P P 17 c$, un dimère $(61 \mathrm{kDa})$ de PP17a, et PP17d (74 kDa) $[5,6]$ (Tableau 1). Par ailleurs, TIP47 présente une forte homologie avec l'adipophiline et les périlipines, protéines associées aux gouttelettes lipidiques (Figure 2). Une action bivalente de TIP47 sur le transport des vésicules contenant le récepteur du M6P d'une part, et celui des gouttelettes lipidiques d'autre part, est discutée dans cet article. 
Dans un souci d'unification entre les termes TIP47 et PP17b, il a été proposé le terme Sandrin (steroid and neutral lipid droplet-associated protein) et leur gène SNDR [7]. Néanmoins, nous utiliserons dans cet article TIP47, terme le plus souvent utilisé dans la littérature.

\section{TIP47 est impliquée dans le recyclage du récepteur du mannose 6-phosphate des endosomes tardifs vers le réseau trans-golgien}

\begin{tabular}{lllcc}
\hline & Poids moléculaire & Spécificité tissulaire & Cancer du col & Placenta \\
PP17a / PP17 & $31,5 \mathrm{kDa}$ & Tissus stéroïdosensibles & ++ & + \\
PP17b / TIP47 & $48 \mathrm{kDa}$ & Ubiquitaire & +++ & +++ \\
PP17c & $60,9 \mathrm{kDa}$ & Tissus stéroïdosensibles & ++ & + \\
PP17d & $74 \mathrm{kDa}$ & Placenta à terme & + & ++ \\
\hline
\end{tabular}

Tableau I. Caractéristiques des différents variants de PP17. Les tissus sensibles aux stéroïdes sont l'utérus, le sein, les testicules. Dans le cancer du col de l'utérus et dans le placenta à terme, une analyse semi-quantitative des protéines PP17 a été réalisée à partir d'études immunohistochimiques (d'après [6]).

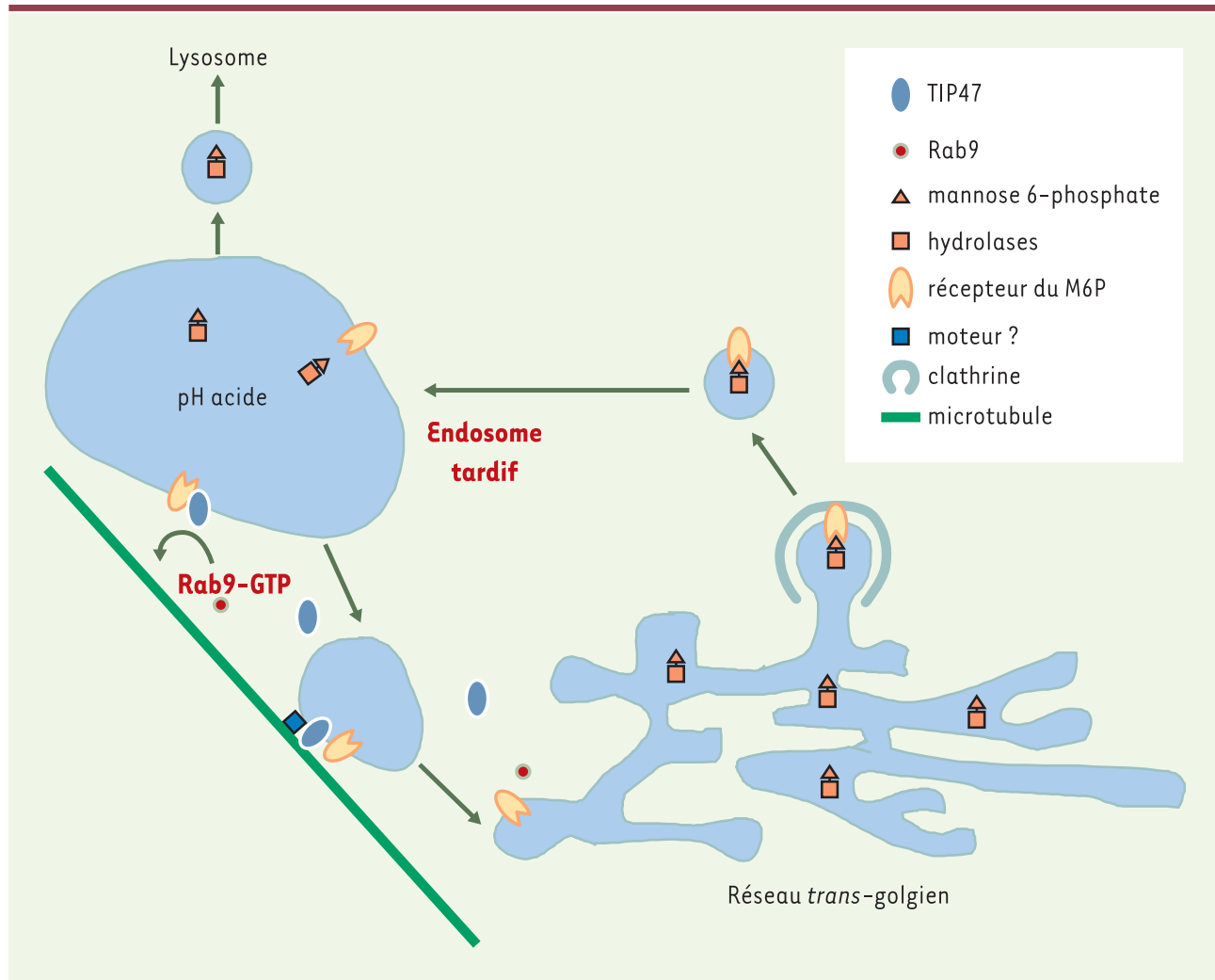

Figure 1. Cycle du récepteur du mannose 6-phosphate. Les hydrolases possédant un résidu terminal mannose-6 phosphate (M6P) sont reconnues par la partie luminale des récepteurs du M6P du réseau trans-golgien. Une vésicule contenant le complexe récepteur-ligand est formée à partir d'un puits recouvert de clathrine, elle perd rapidement son manteau de clathrine et fusionne avec un endosome tardif. Le pH acide local provoque la dissociation du ligand. Les récepteurs peuvent alors être recyclés vers le réseau trans-golgien par un mécanisme qui fait intervenir TIP47 et Rab9GTP. La fraction de TIP47 associée aux membranes ne représente que $20 \%$ du total de la protéine contenu dans la cellule. Le reste est présent dans le cytosol sous forme d'oligomères d'un poids moléculaire de l'ordre de $300 \mathrm{kDa}$. Cette oligomérisation (induite par un domaine situé dans la partie aminoterminale de la protéine, acides aminés 1 à 133) ne serait pas utile à la reconnaissance du récepteur du M6P, mais interviendrait dans la formation d'un manteau protéique (baptisé rétromère) nécessaire au transport du récepteur des endosomes vers le réseau trans-golgien [37-39].
Plusieurs observations indiquent que TIP47 joue un rôle actif dans le recyclage des récepteurs du M6P (Figure 1). TIP47 et le récepteur $\mathrm{Cl}$ du M6P sont tous deux localisés dans les endosomes tardifs et le réseau trans-golgien. De plus, in vitro, un anticorps anti-TIP47 inhibe le retour du récepteur $C D$ du M6P des endosomes vers le réseau trans-golgien. Enfin, la diminution de la concentration intracellulaire de TIP47, induite par des oligonucléotides antisens, provoquent la diminution de la demi-vie du récepteur $\mathrm{Cl}$ du M6P, le transport de ce dernier vers le réseau trans-golgien étant défavorisé, il est adressé vers les lysosomes où il est dégradé [4].

La rétention dans les endosomes du récepteur CD du M6P est essentiellement assurée par le motif Phe-Trp porté par son domaine cytoplasmique, la mutation de ce motif conduisant à l'adressage du récepteur vers les lysosomes [8]. Ce motif PheTrp est en fait responsable de la fixation de TIP47 sur le récepteur $[4,9]$. Le rôle d'un doublet d'acides aminés aromatiques comme étiquette d'adressage vers le réseau transgolgien a été souligné par G. Blot et al. [10]. Ces auteurs ont montré que le domaine cytoplasmique 
de la glycoprotéine Env du virus HIV-1 contient un motif di-aromatique Tyr-Trp, responsable de son interaction avec TIP47 et nécessaire à son transport entre les endosomes tardifs et le réseau trans-golgien. Dans le cas du récepteur $\mathrm{Cl}$ du M6P, des expériences de mutation-délétion ont permis de mettre en évidence un domaine heptapeptidique de séquence Pro-Pro-AlaPro-Arg-Pro-Gly indispensable à la fixation de TIP47 sur son domaine cytoplasmique [11].

D'autres protéines, en association avec TIP47, sont nécessaires au retour du récepteur du M6P vers le réseau transgolgien. La petite protéine G Rab9 sous sa forme active - couplée à du GTP - forme un complexe ternaire avec TIP47 et le récepteur du M6P, et accroît d'un facteur trois l'affinité de TIP47 pour le récepteur [12]. La fixation de Rab9 a lieu sur un domaine spécifique de TIP47, indépendant de celui qui est utilisé dans I'interaction avec le récepteur du M6P. Cependant, TIP47 n'a aucune activité GTPasique ou d'échange nucléotidique [13-15].

\section{TIP47 est aussi associée aux gouttelettes lipidiques}

N. Wolins et al. ont démontré les premiers que TIP47 cytosolique est recrutée sur les gouttelettes lipidiques formées quand les cellules HeLa sont cultivées en présence d'un milieu riche en acides gras [16]. Ces tra-

PP17b/TIP47 Adipophiline Périlipine vaux ont déclenché une vigoureuse polémique avec l'équipe de S. Pfeffer [17], mais depuis, l'association de TIP47 au transport des lipides a été confirmée par différentes techniques et dans différents types cellulaires [7, 18, 19]. Deux protéines sont spécifiquement associées aux gouttelettes lipidiques qui sont formées à partir de la membrane du

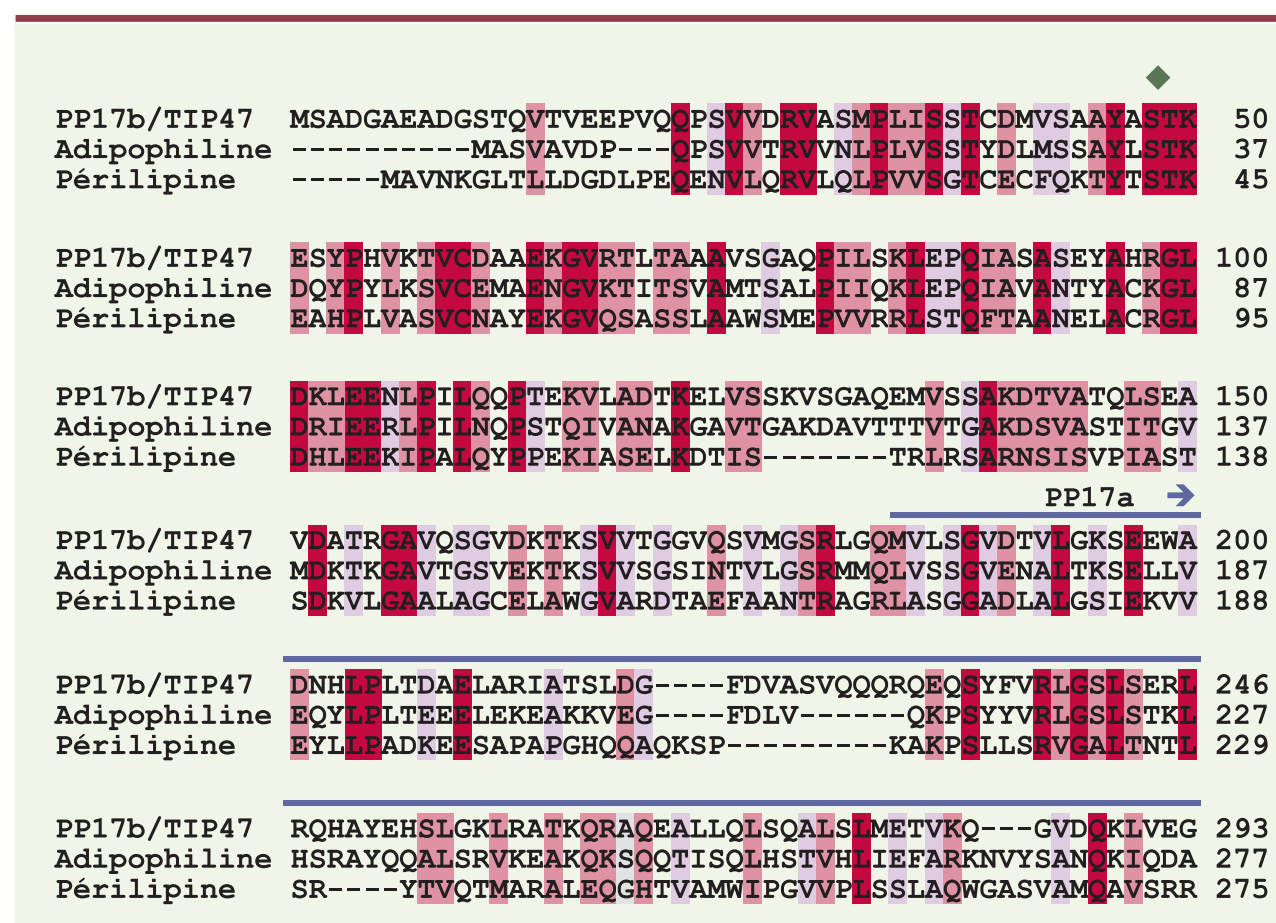

PP17b/TIP47 QEKLHQMWNLWNQKQLQGPEKEPPKPEQVESRALTMFRDIAQQLQATCTS 343 Adipophiline QDKLYLS VEWKRSIGYDDTDESHCA HIESRTLAIARNLTQQLQTTCHT 327 Périlipine RSEVRVPWLHSLAAAQEEDHEDQTDTEGEDTEEEEELETEENKFSEVAAL 325

PP17b/TIP47 LGSS--IQGLPTNVKDQVQQARRQVEDLQATFSSIHS--FQDLSSSILAQ 389 Adipophiline LLSN--IQGVPQNIQDQAKHMGVMAGDIYSVFRNAAS--FKEVSDSLLTS 373 Périlipine PGPRGLLGGVAHTLQKTLQTTISAVTWAPAAVLGMAGRVLHLTPAPAVSS 375

PP17b/TIP47 SRERVASAREAIDHMVEYVAONTPVTWLVG--PFAPGIT---------- 426 Adipophiline SKGQLOKMKESLDDVMDYLVNNTPLNWLVG--PFYPQLTESONAODQGAE 421 Périlipine TKGRAMSLSDA KGVTDNVVDTVVHYVPLPRLSLMEPESEFRDIDNPPAE 425 - PP17a

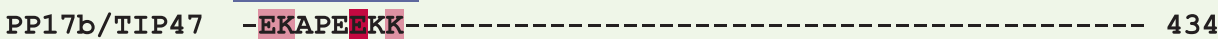

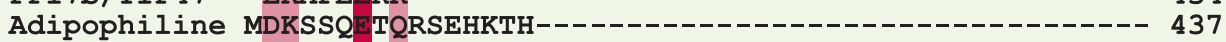
Périlipine VERREA RRASGAPSAGPEPAPRLAQPRRSLRSAQSPGAPPGPGLEDEVA 475

TPAAPRPGFPAVPREKPKRRVSDSFFRPSVMEPIVGRTHYSQLRKKS

\section{2}

Figure 2. Alignement global des séquences primaires humaines de la protéine TIP47, de l'adipophiline et de la périlipine. Le programme d'alignement de séquences ClustalW [40] a été utilisé sur le serveur de l'European Bioinformatics Institute (Hinxton, Royaume-Uni). Les acides aminés identiques sont sur fond rouge. Les symboles sur fonds rose et violet indiquent les substitutions de résidus qui n'entraînent pas (rose) ou peu (violet) de modifications des propriétés physico-chimiques. La séquence de la PP17a, contenue dans celle de TIP47 (M183K434), est surlignée par un trait bleu foncé. Le losange vert au-dessus de la sérine 48 de TIP47 indique un site de phosphorylation potentiel (protéine kinase C et/ou caséine kinase de type II) présent dans les trois protéines et qui est aussi conservé entre les espèces. On ignore encore si TIP47 existe sous une forme phosphorylée. 
réticulum endoplasmique, l'adipophiline (aussi appelée ADRP, adipose differentiation-related protein) et les périlipines [20] (Figure 3). La comparaison de la séquence de TIP47 avec les bases de données de séquences protéiques a révélé l'existence d'une très forte homologie avec celle de l'adipophiline et des périlipines ( $45 \%$ d'homologie avec, dans la partie aminoterminale, $60 \%$ de résidus identiques et $80 \%$ de résidus similaires), qui permet de supposer que ces trois protéines pourraient dériver d'un ancêtre commun [16] (Figure 2). Les périlipines, I'adi- pophiline, TIP47, les protéines de la drosophile (LSD-1 et -2) et de Dictyostelium (LSD1) et la protéine adipocytaire S3-12, ont été regroupées dans la famille des PAT (périlipine-adipophilineTIP47) [21, 22]. Deux sous-domaines ont pu être caractérisés: PAT-1 qui correspond à la partie amino-terminale de ces protéines et qui présente le plus haut degré d'homologie, et PAT-2 qui correspond à la partie plus distale. II faut noter cependant que des protéines connues pour s'associer également avec les gouttelettes lipidiques telles que les oléosines, les cavéolines et

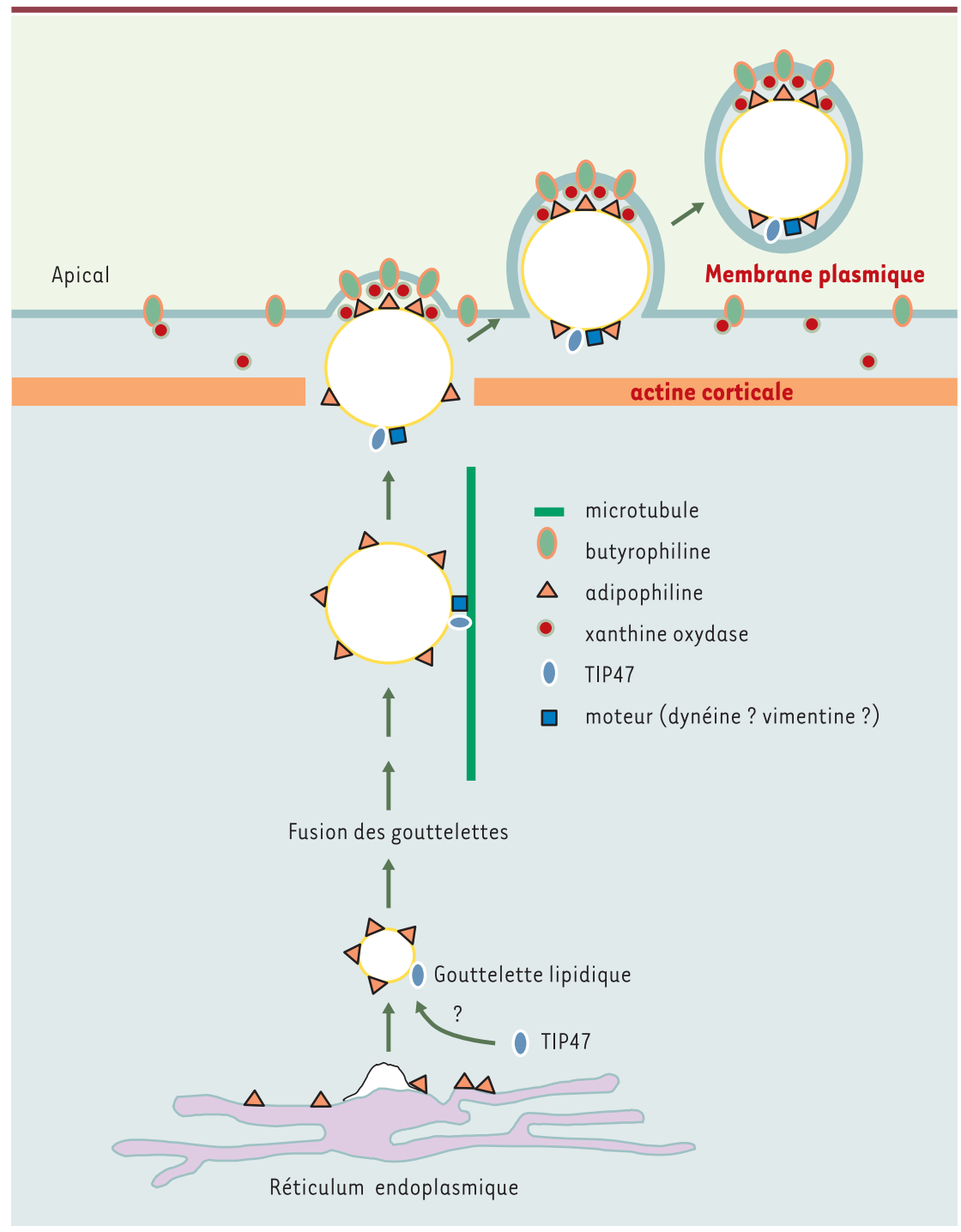

Figure 3. Modèle du mécanisme de formation des gouttelettes lipidiques: exemple de la cellule épithéliale mammaire en lactation. Les gouttelettes lipidiques sont formées à partir de la membrane du réticulum endoplasmique suivant un mécanisme encore très mal connu. Elles sont constituées d'un noyau de tri-acylglycérol et d'esters de cholestérol, entouré par une monocouche de phospholipides. Lors de leur formation, alors que les protéines membranaires spécifiques du réticulum endoplasmique sont exclues de la surface des gouttelettes lipidiques, d'autres telles que l'adipophiline y sont associées [21]. Cette dernière est présente dans tous les types cellulaires. Elle est remplacée par les périlipines dans les adipocytes matures et les cellules stéroïdogéniques. TIP47 serait recrutée à partir du cytosol suivant une chronologie et une régulation qui reste à définir. Le devenir des gouttelettes lipidiques a été particulièrement bien étudié dans la cellule épithéliale mammaire en lactation [41]. Sitôt synthétisées au niveau du réticulum endoplasmique, les gouttelettes lipidiques fusionnent pour former un globule lipidique cytoplasmique. Ce dernier serait transporté sur les microtubules en direction du pole apical de le cellule grâce à la dynéine [42], sous l'éventuel contrôle de la vimentine et de TIP47. Après détachement du microtubule et franchissement du réseau sous-membranaire d'actine grâce à la gelsoline [25], le globule lipidique cytoplasmique s'arrime à la face interne de la membrane apicale de la cellule grâce à la formation d'un complexe ternaire comprenant, dans un rapport molaire 3:5:1, l'adipophiline, la butyrophiline (une protéine transmembranaire) et la xanthine oxydoréductase (une protéine soluble). Au moins six petites protéines $\mathrm{G}$ sont associées à la membrane du globule lipidique, dont quatre semblent être fortement associées avec la butyrophiline et la xanthine oxydoréductase [43]. On ignore si l'adipophiline interagit avec l'une des six petites protéines $G$ et si Rab9 fait partie du groupe. De plus, le rôle de ces petites protéines $G$ dans le processus de formation et d'excrétion des globules lipidiques reste à préciser. Enfin, I'association de TIP47 avec les globules lipidiques du lait laisse supposer que cette protéine n'est pas recyclée [7]. 
les synucléines, ne possèdent pas de domaine PAT. De fait, ces domaines ne semblent pas nécessaires à l'adressage de l'adipophiline vers les gouttelettes lipidiques. En effet, la délétion du domaine PAT-1 ou PAT-2 de l'adipophiline ne modifie ni l'affinité de cette protéine vis-à-vis des gouttelettes lipidiques, ni le nombre de gouttelettes lipidiques contenues dans le cytoplasme [23].

\section{TIP47 serait-elle un régulateur commun de transport?}

Qu'elle soit associée au récepteur du M6P ou aux gouttelettes lipidiques, TIP47 est impliquée dans les structures dynamiques de transport que sont les vésicules et les globules. Dans l'état actuel de nos connaissances, le point commun le plus probable entre ces structures serait le support de leurs mouvements, le cytosquelette. Plusieurs observations étayent cette hypothèse: (1) I'utilisation de Rab9-GFP a permis d'observer que les endosomes présentent une mobilité bidirectionnelle sur les microtubules et de visualiser le retour du récepteur du M6P vers le réseau trans-golgien via des vésicules [13] ; (2) un certain nombre de travaux effectués sur la cellule mammaire en lactation suggèrent un transport guidé au moyen de la dynéine le long des microtubules des gouttelettes lipidiques et/ou des globules lipidiques produits dans la voie de synthèse-sécrétion des lipides du lait tout comme pour le transport des granules de zymogène dans le cas des cellules acineuses du pancréas [24, 25]; (3) les gouttelettes lipidiques et les globules lipidiques possèdent de la dynéine et divers autres moteurs moléculaires à leur périphérie [25]; (4) certaines gouttelettes lipidiques présentent à leur surface de la vimentine, connue pour interagir avec la dynéine cytoplasmique [26-28]; (5) la colchicine, puissant inhibiteur de la formation des microtubules, bloque la sécrétion des constituants du lait in vivo [29]. Cependant, des expériences in vitro suggèrent que toutes les étapes du transport intracellulaire des gouttelettes lipidiques ne sont pas dépendantes des microtubules [30].

Qu'en est-il des autres structures du cytosquelette, les câbles d'actine, les filaments intermédiaires et les protéines contractiles? Si la présence de $\beta$-actine à la périphérie des gouttelettes lipidiques de cellules de la médullosurrénale et d'adipocytes de rat [31], de vimentine, de gelsoline et de géphyrine dans les globules lipidiques du lait a été montré [25], nous connaissons mal le rôle de ces protéines dans le transport intracellulaire des gouttelettes lipidiques et des globules lipidiques.

Quoi qu'il en soit, ces données laissent supposer que l'adipophiline, et/ou TIP47, sont en interaction avec un (des) moteur(s) moléculaire(s) porté(s) par la vésicule contenant le récepteur du M6P ou par les gouttelettes lipidiques pour contrôler leur transport sur le réseau cytosquelettique.

\section{TIP47 et cancer}

TIP47 semble impliquée dans les mécanismes moléculaires de la cancérisation. En effet, dans les carcinomes du col de I'utérus, TIP47 sert de marqueur de l'évolution de la maladie. Cette surexpression de TIP47 dans les carcinomes du col de l'utérus serait liée à l'infection par le virus herpès simplex 2 (HSV-2) car la glycoprotéine virale gD, qui est essentielle à l'entrée du virus dans la cellule, comporte un résidu M6P et se lie au récepteur du M6P pour être adressée vers les endosomes [32]. Cependant, si l'interaction entre la protéine gD et les deux types de récepteur du M6P est bien décrite, ni les perturbations du trafic des récepteurs induites par l'infection virale et/ou celui de la protéine $g D$, ni le rôle joué par TIP47 dans ce phénomène n'ont encore été étudiés. De même, le mécanisme responsable de la présence de TIP47 dans le sang de femmes soit atteintes d'un carcinome du col de l'utérus, soit enceintes (la protéine est détectable dans le sang dès la $7^{\mathrm{e}}$ semaine de gestation, avec un pic vers la $33-34^{e}$ semaine), n'a pas été déterminé. Par ailleurs, TIP47 est surexprimé dans les cellules tumorales isolées de cancers du sein pris à un stade invasif avancé [33], ce qui est en accord avec les nombreuses données démontrant l'importance du récepteur du M6P dans le cancer du sein et l'existence d'un lien direct entre les modifications du métabolisme des lipides et le développement de ces cancers [34].

\section{Conclusions}

TIP47 se trouve être impliquée simultanément dans deux fonctions cellulaires différentes, le trafic du récepteur du mannose 6-phosphate et celui des gouttelettes lipidiques cytoplasmiques. À ce jour, il n'y a pas de lien direct connu entre ces deux fonctions. De même, alors que le récepteur $\mathrm{Cl}$ du M6P est identique à $100 \%$ au récepteur de I'IGF II, le lien entre ces deux fonctions reste inconnu [35]. Le rôle grandissant dans le fonctionnement cellulaire de protéines multifonctionnelles appelées moonlighting proteins (to moonlight: travailler au noir) met de plus en plus à mal le dogme « une protéine = une fonction », et la multifonctionnalité protéique apparaît de moins en moins être l'exception [36]. L'élucidation des liens de causalité entre les diverses fonctions du récepteur du M6P et de TIP47 sera un défi majeur des années à venir. $\diamond$

\section{SUMMARY}

The double-play of PP17/TIP47

A missing link in the understanding of the mechanisms of transport of the mannose 6-phosphate receptors has recently been discovered, following the identification of the protein TIP47. In association with Rab9-GTP, this protein is responsible for the 
return of the receptors from the late endosomes back to the trans-Golgi network. Curiously, the same protein called PP17b, was described as a placental protein twenty years ago, and more recently, as a blood marker for human uterine cervical cancer. The sequence of PP17b/TIP47 displays not only a strong homology with those of adipophilin and the perilipins, two proteins known to be involved in the intracellular traffic of lipid droplets but also PP17b/TIP47 is associated with the later. How this ubiquitous protein could participate in processes as different as the mannose 6-phosphate receptors traffic and the formation and/or traffic of lipid droplets? A tentative hypothesis is put forward. $\bullet$

\section{RÉFÉRENCES}

1. Méresse S, Bauer U, Ludwig T, Schmidt A, Hoflack B. Bases moléculaires du transport vers les lysosomes. Med Sci (Paris) 1993; 9 : 148-56.

2. Hille-Rehfeld A. Mannose 6-phosphate receptors in sorting and transport of lysosomal enzymes. Biochim Biophys Acta 1995; 1241 : 177-94.

3. Kornfeld S. Structure and function of the mannose 6-phosphate/insulinlike growth factor II receptors. Annu Rev Biochem 1992 ; 61 : 307-30.

4. Diaz $\varepsilon$, Pfeffer SR. TIP47: a cargo selection device for mannose 6-phosphate receptor trafficking. Cell 1998; 93: 433-43.

5. Bohn $\mathrm{H}$, Kraus W, Winckler W. Purification and characterization of two new soluble placental tissue proteins (PP13 and PP17). Oncodev Biol Med 1983; 4: 343-50.

6. Than NG, Sumegi $B$, Than GN, et al. Cloning and sequence analysis of cDNAs encoding human placental tissue protein 17 (PP17) variants. Eur J Biochem 1998; 258: $752-7$.

7. Than NG, Sumegi B, Bellyei S, et al. Lipid droplet and milk lipid globule membrane associated placental protein 17b (PP17b) is involved in apoptotic and differentiation processes of human epithelial cervical carcinoma cells. Eur J Biochem 2003; 270: 1176-88.

8. Schweizer A, Kornfeld S, Rohrer, J. Proper sorting of the cation-dependent mannose 6-phosphate receptor in endosomes depends on a pair of aromatic amino acids in its cytoplasmic tail. Proc Natl Acad Sci USA 1997; 94 : 14471-6.

9. Nair P, Schaub BE, Rohrer J. Characterization of the endosomal sorting signal of the cation-dependent mannose 6-phosphate receptor. J Biol Chem 2003; $278: 24753-8$.

10. Blot $G$, Janvier $K$, Le Panse $S$, et al. Targeting of the human immunodeficiency virus type 1 envelope to the trans-Golgi network through binding to TIP47 is required for Env incorporation into virions and infectivity. J Virol 2003; 77: 6931-45.

11. Orsel JG, Sincock PM, Krise JP, Pfeffer SR. Recognition of the 300-kDa mannose 6-phosphate receptor cytoplasmic domain by 47-kDa tail-interacting protein. Proc Natl Acad Sci USA 2000; 97 : 9047-51.

12. Lombardi $D$, Soldati $T$, Riederer $M A$, et al. Rab9 functions in transport between late endosomes and the trans-Golgi network. EMBOJ 1993; 12: 677-82.

13. Barbero P, Bittova L, Pfeffer SR. Visualization of Rab9-mediated vesicle transport from endosomes to the trans-Golgi in living cells. J Cell Biol 2002; 156: 511-8.

14. Carroll KS, Hanna J, Simon I, et al. Role of Rab9 GTPase in facilitating receptor recruitment by TIP47. Science 2001 ; 292: 1373-6.

15. Hanna J, Carroll K, Pfeffer SR. Identification of residues in TIP47 essential for Rab9 binding. Proc Natl Acad Sci USA 2002; 99 : 7450-4.

16. Wolins NE, Rubin B, Brasaemle DL. TIP47 associates with lipid droplets. J Biol Chem $2001 ; 276: 5101-8$.

17. Barbero P, Buell $\varepsilon$, Zulley S, Pfeffer SR. TIP47 is not a component of lipid droplets. J Biol Chem 2001; 276: 24348-51.

18. Miura S, Gan JW, Brzostowski J, et al. Functional conservation for lipid storage droplet association among perilipin, ADRP, and TIP47 (PAT)-related proteins in mammals, Drosophila, and Dictyostelium. J Biol Chem 2002; 277 : 32253-7.

19. Ohashi M, Mizushima N, Kabeya Y, Yoshimori T. Localization of mammalian NAD (P)H steroid dehydrogenase-like protein on lipid droplets. J Biol Chem 2003; 278: 36819-29.
20. Murphy DJ, Vance J. Mechanisms of lipid-body formation. Trends Biochem Sci 1999 ; $24: 109-15$

21. Lu X, Gruia-Gray J, Copeland NG, et al. The murine perilipin gene: the lipid droplet-associated perilipins derive from tissue-specific, mRNA splice variants and define a gene family of ancient origin. Mamm Genome 2001 ; 12 : 741-9.

22. Wolins NE, Skinner JR, Schoenfish MJ, et al. Adipocyte protein S3-12 coats nascent lipid droplets. J Biol Chem 2003 ; 278 : 37713-21.

23. McManaman JL, Zabaronick W, Schaack J, Orlicky DJ. Lipid droplet targeting domains of adipophilin. J Lipid Res $2003 ; 44: 668-73$.

24. Kraemer J, Schmitz F, Drenckhahn D. Cytoplasmic dynein and dynactin as likely candidates for microtubule-dependent apical targeting of pancreatic zymogen granules. Eur J Cell Biol $1999 ; 78: 265-77$.

25. Wu CC, Howell KE, Neville MC, et al. Proteomics reveal a link between the endoplasmic reticulum and lipid secretory mechanisms in mammary epithelial cells. Electrophoresis $2000 ; 21: 3470-82$.

26. Franke WW, Hergt M, Grund C. Rearrangement of the vimentin cytoskeleton during adipose conversion: formation of an intermediate filament cage around lipid globules. Cell $1987 ; 49$ : 131-41.

27. Helfand BT, Mikami A, Vallee RB, Goldman RD. A requirement for cytoplasmic dynein and dynactin in intermediate filament network assembly and organization. J Cell Biol $2002 ; 157: 795-806$.

28. McGookey DJ, Anderson RG. Morphological characterization of the cholesteryl ester cycle in cultured mouse macrophage foam cells.

J Cell Biol 1983; 97 : 1156-68.

29. Knudson CM, Stemberger BH, Patton S. Effects of colchicine on ultrastructure of the lactating mammary cell: membrane involvement and stress on the Golgi apparatus. Cell Tissue Res 1978 ; 195 : 169-81.

30. Daudet F, Augeron C, Ollivier-Bousquet M. Early action of colchicine, ammonium chloride and prolactin, on secretion of milk lipids in the lactating mammary gland. EurJ Cell Biol 1981; 24 : 197-202.

31. Fong $\mathrm{TH}, \mathrm{Wu} \mathrm{CH}$, Liao, et al. Association of globular beta-actin with intracellular lipid droplets in rat adrenocortical cells and adipocytes. Biochem Biophys Res Commun $2001 ; 289$ : 1168-74.

32. Brunetti CR, Burke RL, Kornfeld $S$, et al. Herpes simplex virus glycoprotein D acquires mannose 6-phosphate residues and binds to mannose 6-phosphate receptors. J Biol Chem $1994 ; 269$ : 17067-74.

33. Mellick AS, Day CJ, Weinstein SR, et al. Differential gene expression in breast cancer cell lines and stroma-tumor differences in microdissected breast cancer biopsies revealed by display array analysis. Int J Cancer 2002 ; 100 : 172-80.

34. Stoll BA. Upper abdominal obesity, insulin resistance and breast cancer risk. Int J Obes Relat Metab Disord $2002 ; 26: 747-53$.

35. McDonald RG, Pfeffer SR, Coussens L, et al. A single receptor binds both insulin-like growth factor II and mannose-6-phosphate. Science 1988; 239 : 1134-7.

36. Jeffery CJ. Moonlighting proteins. Trends Biochem Sci $1999 ; 24: 8-11$.

37. Barlowe C. Traffic COPs of the early secretory pathway. Traffic 2000 ; $1: 371-7$.

38. Pfeffer SR. Membrane transport: retromer to the rescue. Curr Biol 2001 ; 11 : R109-11.

39. Sincock PM, Ganley IG, Krise JP, et al. Self-assembly is important for TIP47 function in mannose 6-phosphate receptor transport. Traffic $2003 ; 4: 18-25$.

40. Thompson JD, Higgins DG, Gibson TJ. ClustalW improving the sensitivity of progressive multiple sequence alignment through sequence weighting, positionspecific gap penalties and weight matrix choice. Nucleic Acids Res 1994 ; $22: 4673-80$.

41. Mather IH, Keenan TW. Origin and secretion of milk lipids. J Mammary Gland Biol Neoplasia $1998 ; 3: 259-73$.

42. Lacey ML, Haimo LT. Cytoplasmic dynein binds to phospholipid vesicles. Cell Motil Cytoskeleton $1994 ; 28: 205-12$.

43. Ghosal D, Ankrapp D, Keenan TW. Low molecular mass GTP-binding proteins are secreted from mammary epithelial cells in association with lipid globules. Biochim Biophys Acta 1993 ; 1168 : 299-306.
TIRÉS À PART

A. Pauloin 